\title{
RareNoise: non-equilibrium effects in detectors of gravitational waves
}

\author{
L Conti ${ }^{1}, M$ Bonaldi ${ }^{2}$ and L Rondoni ${ }^{3}$ \\ 1 INFN, Sezione di Padova, Via Marzolo 8, I-35131 Padova, Italy \\ E-mail: Livia.Conti@pd.infn.it \\ 2 Istituto di Fotonica e Nanotecnologie, CNR-Fondazione Bruno Kessler, 38100 \\ Povo, Trento, Italy and INFN, Gruppo Collegato di Trento, Sezione di Padova, \\ 38100 Povo, Trento, Italy \\ 3 Dip. di Matematica, Politecnico di Torino, Corso Duca degli Abruzzi 24, \\ 10129 Torino, Italy and INFN Sezione di Torino, Via Pietro Giuria, 1, I-10125 \\ Torino, Italy
}

\begin{abstract}
The RareNoise project investigates non-equilibrium effects in Gravitational Wave detectors. We illustrate the Physics behind the project and the planned project development, involving experimental, numerical and theoretical research.
\end{abstract}

PACS numbers: 04.80.Nn, 05.70.Ln, 05.40.-a, 07.05.Fb 


\section{Introduction}

Ground based detectors of Gravitational Waves (abbreviated with GW in the following) [1, 2] are very sophisticated experiments, consisting of Michelson laser interferometers with arm length of up to a few kilometers, or mechanical oscillators with masses of up to several tons. They are most sensitive in the frequency band $\left(10 \div 10^{4}\right) \mathrm{Hz}$; depending on the source type, the temporal evolution of GW signals expected in this frequency band changes widely, ranging from quasi-periodic to impulsive $(\lesssim 1 \mathrm{~ms})$ signals. Present detectors are expected to detect occasionally GW signals. Advanced versions of present laser interferometers will be operative from 2013; they will reach a sensitivity close to the quantum limit set by the uncertainty principle and are predicted to detect GW events possibly as often as daily. There is an ongoing theoretical research focusing on the prediction of signal waveforms for a wide class of phenomena that might happen in the universe and that might be detected [2]. In some cases GW emission is expected in connection with different astrophysical signals (such as Gamma Ray Bursts, neutrino bursts, X-rays). Apart from these studies, there is no anticipation or independent trigger on the signal arrival time: therefore the experimentalists try to run the detectors around the clock and look for any rare event, ie any fluctuation of the detector output that is out of the statistics by several standard deviations. In this context the detector quality is not only given in terms of its peak sensitivity but also of its duty cycle and stationarity of its noise: the latter is fundamental to lower the false alarm rate and assure a confident detection.

Making a detector with noise of pure stationary Gaussian statistics has proven to be as much difficult as making a very sensitive one, these two requirements often being contrasting. Up to now, any gravitational wave detector is characterized by noise that is neither stationary nor Gaussian, showing for instance non-Gaussian "tails" in the probability distribution. If these are large, they bias the statistics thus making more difficult the achievement of a given level of statistical confidence [3]. In the past some of these unexpected noise fluctuations have been claimed as true GW signals, falsified by subsequent analysis. Because of the difficulty in distinguishing a true GW signal from a non-modeled noise fluctuation, joint GW searches are mandatory that combine data from multiple detectors with uncorrelated noise. This brings the advantage not only of reducing the false alarm rate, but also of improving the sky coverage, the detection confidence and the estimation of the GW signal parameters.

From the thermodynamic viewpoint GW experiments are usually modeled as equilibrium systems but it is doubtful that this description is justified. For instance, in interferometric detectors the fibers which suspend the interferometer mirrors are driven out of equilibrium by the thermal gradient due to the light power dissipated into the mirror substrate and coating, extracted as heat through the fibers themselves. The instrument is further pushed towards a non-equilibrium state by the thermal compensation technique [4] which aims to overcome the mirror thermal lensing caused by the thermal expansion due to the laser power being absorbed not uniformly: interferometer's mirrors are subject to external, additional thermal load with the purpose of creating a thermal gradient that further deforms the mirror so to get the desired radius of curvature. In detectors based on massive mechanical resonators, temperature gradients which exist under operating conditions (below the superconductive transition) could drive the resonant-mass detector out of equilibrium. Feedback damping of the electromechanical resonators has already proved to cause a non-equilibrium steady-state [5]. Interferometric displacement sensors discussed 
in ref. [6] may also cause thermal gradients due to light absorption on mirror substrates and coatings. Laser-sources and Fabry-Perot optical resonators employed in both interferometric detectors and sensors are non-equilibrium systems. Due to the expected increase in the sensitivity of the next generation of the GW detectors favored also by the use of high laser powers and cryogenic operation, the influence of non-equilibrium effects on the statistics of fluctuations, is expected to rise. As a quantitative example, in the proposed cryogenic interferometric GW detector LCGT the fibers suspending the mirrors have one end attached to the mirrors at $20 \mathrm{~K}$ and the other to a mass at $10 \mathrm{~K}$, with a heat flow of about $1 \mathrm{~W}$ [7]. Similarly a future, 3rd generation European GW interferometric detector [8] might employ cryogenic mirrors with possibly higher thermal gradients and comparable heat flow.

Research of non-equilibrium statistical mechanics is facing a renewed interest: even if a general theory is still lacking, for explaining the physical properties of a macroscopic non-equilibrium system, on the basis of the behavior of its microscopic constituents, several intermediate results have been proven and are collectively known as Fluctuation Theorems [9]. These theorems state a quantitative relation between the probability of observing a certain rate of entropy production or energy dissipation and the probability of observing an equal amount rate of entropy consumption or energy gain. While this result might appear as meager, it has aroused much interest because it stands up as one of the very few exact and parameter-free results obtained so far in non-equilibrium statistical mechanics: furthermore, it has recently been extended to cover a wide variety of phenomena and observables [9, 10]. This theoretical research aims at describing the low probability, large fluctuations in the noise distribution, which are due to the interplay of the external driving, of the material properties and of the dissipation mechanisms. We found that these fluctuations differ from the Gaussian noise affecting equilibrium systems, usually assumed even in the analysis of systems such as ours. So far experimental efforts that test and stimulate these studies are very limited in number and sometimes their interpretation is difficult. Experiments involve mainly small-scale systems as molecular machines and nanostructured materials: in fact in large scale systems the large fluctuations become vanishingly rare. Only recently experiments based on non-equilibrium macroscopic systems have been performed: see for instance ref. [11] for the study of a standard metallic resistor crossed by a steady-state current and ref. [5] for the study of a macroscopic electromechanical oscillator cooled by an active feedback system.

If non-equilibrium effects are important in GW detectors, then the noise statistics is intrinsically different from the equilibrium Gaussian statistics. Large fluctuations are less rare: they increase the false alarm rate and worsen the detector reliability. On the other hand if non-equilibrium effects are demonstrated to be important, the experimental design of GW detectors must be reviewed so to minimize the occurrence of the large noise fluctuations and the theory of non-equilibrium fluctuations used in the data analysis processes. As a consequence, detectors might aim at a more confident detection at a given signal amplitude, or claim detection at a given confidence level but at lower signal amplitudes: in both cases detection chances are increased.

\section{The RareNoise project}

RareNoise [12] is a 5 year project started in July 2008 and funded by the European Research Council. The project is devoted to the study of non-equilibrium fluctuations in macroscopic systems; the study is performed via two mutually reinforcing 
approaches: numerical and analytical analysis and laboratory experiments. First application of the knowledge gained while performing the research is offered by groundbased GW detectors: the research will determine the extent to which detectors of GW are non-equilibrium systems.

The research focuses on the effect of temperature gradients in GW detectors. Situations similar to those that drive out of equilibrium mechanical subsystems of the detectors will be reproduced experimentally on smaller scale devices that can be realized and characterized more easily. The effects will be investigated of temperature gradients on the spontaneous vibration fluctuations of low-loss mechanical oscillators. The effects will be studied in a small frequency range around the oscillator resonance, set to be within the frequency range of interest for the GW detectors. In order to reproduce a situation similar to those experienced in GW detectors, it is mandatory that the oscillator is a very low loss one, ultimately limited by internal loss mechanisms. In contrast to previous experimental works on Fluctuation Theorems, where the system is often surrounded by a viscous fluid, this research focuses on material intrinsic damping, a situation common to high-precision experiments.

Since present theoretical understanding of the non-equilibrium fluctuations is not thorough, it is very difficult to predict how and how much the spontaneous vibrations of a mechanical oscillator under steady-state heat flux differ from those at the thermodynamic equilibrium: the latter are fully known, being distributed with a Gaussian law and quantified by the Fluctuation-Dissipation theorem [13]. A crude application of one of the formulae obtained through the recent extensions of the theory of non-equilibrium fluctuations [9] in macroscopic systems such as in GW detectors leads to a prediction regarding only exaggeratedly large fluctuations.

Since the first GW detectors, two materials have been identified for their low loss properties as best choices for construction of key detector parts: a metal, namely the aluminum alloy A15056, and a semiconductor, namely single-crystal Silicon. The former is known to have intrinsic mechanical losses $\lesssim 3 \times 10^{-8}$ below $10 \mathrm{~K}$ [14]: along with the large availability and low cost, this feature motivates its usage as constituting material of most of the resonant mass, cryogenic GW detectors ever operated and of all running ones. At room temperature the intrinsic mechanical losses of Al5056 are at the level of $3 \times 10^{-6}[14]$. Single crystals of Silicon can reach mechanical losses as low as $5 \times 10^{-10}$ at $3.5 \mathrm{~K}[15]$ and $10^{-8}$ [16] at room temperature. These properties make Silicon the elective material for the next generation of gravitational wave detectors, while already being the choice for most MEMS and high-precision devices.

RareNoise focuses on these two materials, which will be modeled by means of molecular dynamics techniques. Given their different, temperature dependent levels of intrinsic mechanical losses, the experimental work is organized into three consecutive phases:

(i) an initial phase, with the losses expected to be in the range $10^{-3} \div 10^{-6}$. Experiments are performed at equilibrium temperatures of $300 \mathrm{~K}$ and $77 \mathrm{~K}$ with both Al5056 and Silicon. This is referred to as phase 1 in the following;

(ii) an intermediate phase, with the losses expected to be in the range $10^{-6} \div 10^{-7}$. Experiments are performed at the equilibrium temperature of $4.2 \mathrm{~K}$ with both Al5056 and Silicon. This is referred to as phase 2 in the following;

(iii) a final phase, with the losses expected to be in the range $10^{-6} \div 10^{-8}$. Experiments are performed at equilibrium temperatures of $300 \mathrm{~K}, 77 \mathrm{~K}$ only with Silicon. This is referred to as phase 3 in the following. 


\begin{tabular}{|c|c|c|c|}
\hline rod length & $0.1 \mathrm{~m}$ & rod cross-section & $28 \times 10^{-6} \mathrm{~m}^{2}$ \\
rod mass & $0.0078 \mathrm{~kg}$ & $m_{l}$ & $0.208 \mathrm{~kg}$ \\
$m_{l}$ bottom surface & $1.82 \times 10^{-3} \mathrm{~m}^{2}$ & capacitor gap & $44 \mu \mathrm{m}$ \\
IR source support mass & $0.58 \mathrm{~kg}$ & oscillator assembly mass & $3.6 \mathrm{~kg}$ \\
\hline
\end{tabular}

Table 1. Main figures of the Phase 1 prototype oscillator in Al5056.

\subsection{Phase 1}

Phase 1 will give first indications regarding the effects of thermal gradients on the spontaneous fluctuations of mechanical oscillators and therefore has a broad spectrum of investigation. Experiments are devoted to the study of the longitudinal and transverse vibration of oscillators when a thermal gradient is applied between the longitudinal extremes of the body. The study of transverse vibrations has direct application to the GW interferometers since it mimics the motion of the fibers supporting the mirrors, which will be subject to longitudinal thermal gradients; moreover it constitutes a new challenge for theoretical models that so far have not considered such a situation. After the room temperature campaign is completed, the first experimental results are expected: they will be compared with theoretical knowledge and used to develop the appropriate theoretical models. Then, the measurements will be repeated at the equilibrium temperature of $77 \mathrm{~K}$ : the temperature variation brings about material properties changes. Therefore it constitutes a unique training ground for developing a sound theoretical model of the spontaneous vibrations of an elastic body subject to thermal gradients. In particular it will be interesting to investigate the effect of varying the thermal expansion coefficient of the oscillator on the statistical properties of the non-equilibrium fluctuations: in this regard Silicon turns out to be of major interest since its coefficient of thermal expansion changes sign both at $124 \mathrm{~K}$ and $18 \mathrm{~K}$.

A prototype of the Phase 1 Al5056 oscillator has already been realized and successfully tested. The oscillator consists of a rod with cross cross-section fixed at one end and loaded by a mass (referred to as $m_{l}$ in the following) at the other end: the oscillator is kept with the rod along the vertical and the load mass at the bottom. The oscillator was designed with the aid of Finite Element Modeling (FEM) analysis so that its first longitudinal mode occurs at about $1.5 \mathrm{kHz}$ while the first flexural modes occur at $317 \mathrm{~Hz}$ and $490 \mathrm{~Hz}$. In figure 1 and figure 2 we show respectively the deformations corresponding to the first longitudinal mode and the second flexural mode, with respect to the shape of the oscillator at rest, as resulting from the FEM analysis. Main figures of the oscillator are listed in table 1.

The vibrations of the oscillator load mass are measured by a capacitive readout coupled to a low noise amplifier. The capacitive sensor is realized by facing an aluminum plate to the bottom surface of the load mass (see figure 3): the capacitor is thus realized between the mass and the facing plate. The capacitive sensor works in a constant charge configuration, once it is biased up to $500 \mathrm{~V}$. The gap between the plate and $m_{l}$ is realized by a $50 \mu \mathrm{m}$ teflon spacer: this is supported by two arms that extends along the vertical direction at two sides of the rod. The facing surfaces of the oscillator, of the plate supports and of the plate are finished via diamond turning so that a static electric field of about $1 \times 10^{7} \mathrm{MV} / \mathrm{m}$ can be applied. Using a commercial, low noise amplifier with noise voltage at the level of a few $n \mathrm{~V} / \sqrt{\mathrm{Hz}}$ (corresponding to a few $10^{-16} \mathrm{~m} / \sqrt{\mathrm{Hz}}$ ), the oscillator resonance excited by the equilibrium thermal 
noise is expected to prevail in a frequency bandwidth of about $10 \mathrm{~Hz}$, assuming losses at the level of $4 \times 10^{-4}$ at room temperature. At room temperature, in order to realize the thermal gradient along the oscillator, and thus to set the oscillator in a non-equilibrium steady-state, we make use of a thermal control system that stabilizes the temperature of the rod top end and that of the mass $m_{l}$ : the control acts on Peltier cells to cool the temperature of the rod top end while the rod load mass $m_{l}$ is heated via radiative heat exchange by an infrared thermal source which is faced to it (see figure 3). Being non-contacting, the radiative heating guarantees that no external losses are added to the oscillator. The temperature of $m_{l}$ is measured by a no-touching sensor, ie a IR thermometer faced to the mass (see figure 3). To improve the radiative coupling with both IR source and thermometer, the portion of the $m_{l}$ mass that is

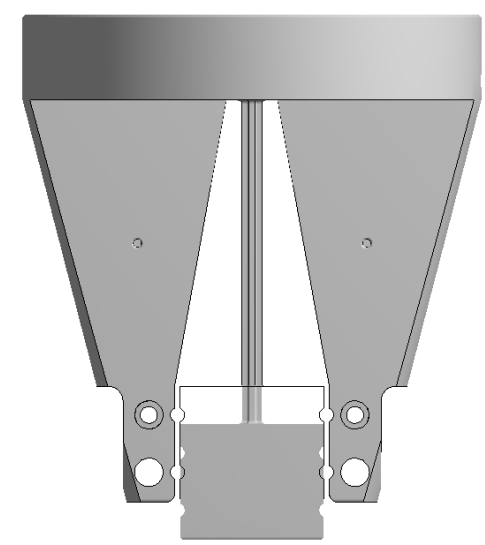

Figure 1. Deformation of the oscillator assembly in correspondence of the first longitudinal mode of the mechanical oscillator, as predicted by the FEM analysis: the mode resonates at $1.5 \mathrm{kHz}$. The shape of the oscillator at rest is shown as black solid lines for comparison.

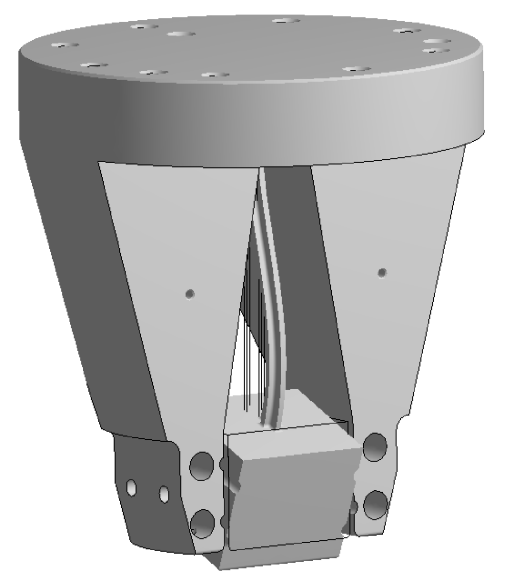

Figure 2. Deformation of the oscillator assembly in correspondence of the second flexural mode of the mechanical oscillator, as predicted by the FEM analysis: the mode resonates at $490 \mathrm{~Hz}$. The shape of the oscillator at rest is shown as black solid lines for comparison. 
facing to them is hard anodized. A temperature gradient of about $(10-30) \mathrm{K}$ will be applied at room temperature. Figure 3 shows the assembly of the oscillator, along with the capacitive sensor, the IR source and the thermopile. In figure 4 we show a picture of the Phase 1 prototype of Al5056 mechanical oscillator where the two thermometers used for the control of the thermal gradient are clearly visible.

\subsection{Phase 2}

The oscillators of phase 1 will be studied at the equilibrium temperature of $4.2 \mathrm{~K}$ : not only this offers the advantage of different material properties, in particular minimum losses, but also eases the establishment of larger relative thermal gradients. Given the lower temperature, this phase is more demanding on the vibration sensors both in terms of sensitivity and working environment: only the degrees of freedom will be monitored that can be adequately measured.

\subsection{Phase 3}

Phase 3 will concentrate on Silicon: a specially designed, monolithic oscillator will be produced to profit from the very low intrinsic losses of this material in the full temperature range from $300 \mathrm{~K}$ to $4.2 \mathrm{~K}$. This will constitute the experimental situation most similar to interferometric GW detectors of third generation: therefore

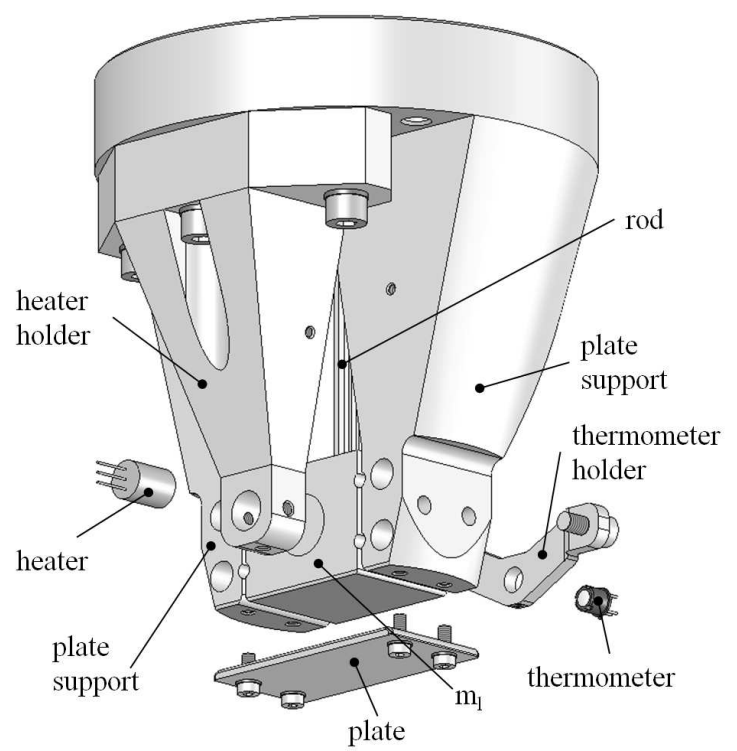

Figure 3. Exploded view of the assembly of the Phase 1 Al5056 oscillator. The oscillator is machined from a single piece along with the supports for the second armature of the capacitor: the oscillator consists of a rod with cross cross-section with one end fixed and one end loaded by mass $m_{l}$. A $50 \mu \mathrm{m}$ thick Teflon spacer is interposed between the plate and its supports to realize the gap of the capacitive sensor. The plate supports hold also the IR thermometer that is faced to a hard anodized, $\sim 0.02 \mathrm{~m}$ in diameter spot on the mass $m_{l}$. On the other side of the IR thermometer with respect to $m_{l}$, a support holds the IR source in place, in front of a similar anodized spot on the mass $m_{l}$. 
quantitative indications about non-equilibrium effects in such instruments are expected as final result, along with refinements of theoretical predictions. At present the experiments for phase 3 are harder to plan than those of phases 1 and 2: for instance, decision on the kind of acoustic mode (whether longitudinal or transverse, given a longitudinal thermal gradient) to be monitored will be taken on the basis of the experimental and theoretical results of previous research.

\subsection{Theoretical and numerical work}

The theoretical work will be developed in parallel with the experiments, in order to identify the models and the parameters which can most realistically simulate the behavior of the experimental setup. Initially both mathematical and numerical investigations will be pursued of 1-dimensional chains of oscillators, on which a large literature exists, and which have recently found application in, e.g. the study of heat transport in carbon nanotubes [17]. Then, generalizations of such models, in

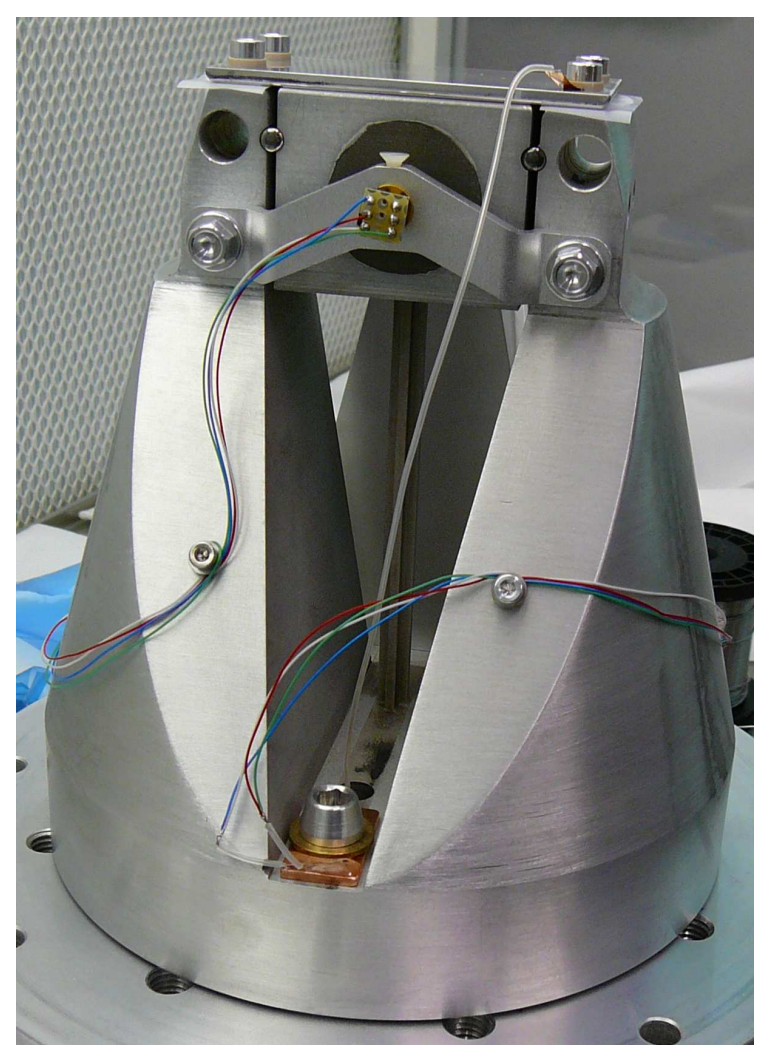

Figure 4. Photograph showing the Phase 1 Al5056 prototype oscillator. At the base of the rod a NTC thermistor is mounted on a copper plate: this is the thermometer used in the control loop to stabilize the temperature of the rod base. The Teflon spacers and the cable carrying the capacitive sensor signal toward its preamplifier is clearly seen originating from the plate. The IR thermometer is also clearly visibile, facing an anodized spot on the mass $m_{l}$. In this photo the oscillator is kept upside-down, with the mass $m_{l}$ at the top; moreover the mass is held in place by two spacers which are removed once the oscillator is reversed. 
which several chains will be coupled to go beyond the 1-dimensional cases will be simulated by means of non-equilibrium molecular dynamics techniques. Particular attention will be devoted to the construction of the particles interaction potentials, in order to approximate as closely as possible the behavior of real rods, in the range of parameters accessible to experimental tests. Once the models are sufficiently refined, that they perform adequately in a range of parameters in which direct comparison with experiment is possible, they will be used to compute the observables which cannot be experimentally tested at the lowest temperatures, but which are still useful in the characterization of the non-equilibrium state of the system, and of its noise in particular. The work will end with refinements of the non-equilibrium theory, in particular by assessing the validity of the normal mode expansion formalism, and with novel applications to the interferometric GW detectors: non-equilibrium fluctuations will be estimated and their effect on the detection capabilities will be clarified.

In high-precision experiments, the sensitivity is often limited by the mechanical thermal noise: since at equilibrium any displacement of an elastic body can be expanded on the basis formed by the acoustic normal modes, the mechanical thermal noise can be modeled as the superposition of noise contributions from each acoustic mode [18]. It is actually matter of research of RareNoise to understand the extent to which the normal mode formalism is valid in non-equilibrium situations: this is crucial for a correct interpretation of the experimental data.

\section{Acknowledgments}

We thank G. A. Prodi for useful discussions. The research leading to these results has received funding from the European Research Council under the European Community's Seventh Framework Programme (FP7/2007-2013) / ERC grant agreement n 202680. The EC is not liable for any use that can be made on the information contained herein.

\section{References}

[1] Whitcomb S E 2008 Class. Quantum Grav. 25114013

[2] See for instance: Sathyaprakash B S and Schutz B F 2009 Liv Rev Rel 12 , online at www.livingreviews.org/lrr-2009-2

[3] Allen A 2005 Phys. Rev. B 71062001

[4] Hello P 2001 Eur. Phys. J. D 15 373-383

[5] Bonaldi M et al 2009 Phys. Rev. Lett. 103010601

[6] Conti L et al 1998 Rev. Sci. Instrum. 69 554-558

[7] Tomaru T et al 2002 Phys. Lett. A 301 215-219

[8] www.et-gw.eu

[9] See for instance: Evans D J, Cohen E G D and Morriss G P 2003 Phys. Rev. Lett. 71 2401; Rondoni L and Mejia-Monasterio C 2007 Nonlinearity 20 R1-R37 and refs therein; Marini Bettolo Marconi U, Puglisi A, Rondoni L and Vulpiani A 2008 Phys. Rep. 461 111-195

[10] Gallavotti G 2007 arXiv:cond-mat/0701124; Puglisi A, Rondoni L and Vulpiani A $2006 \mathrm{~J}$. Stat. Mech. P08010

[11] Garnier N and Ciliberto S 2005 Phys. Rev. E 71060101

[12] www.rarenoise.lnl.infn.it

[13] Callen B H and Welton T H 1951 Phys. Rev. 83 34-40

[14] Coccia E and Niinikoski T O 1984 Lett. Nuovo Cim. 41242

[15] McGuigan D F 1978 Journ. Low Temp. Phys. 30621

[16] Numata K et al 2001 Phys. Lett. A 284 162-171

[17] Livi R and Lepri S 2003 Nature 23 January 327

[18] See for instance: Saulson P R 1990 Phys. Rev. D 422437 